\title{
Clinical Observation and Therapeutic Evaluation of Rh- endostatin Combined with DP Regimen in Treating Patients with Advanced Esophageal Cancer
}

\author{
Wen-Ying Deng ${ }^{1}$, Tao Song ${ }^{2}$, Ning $\mathrm{Li}^{1}$, Su-Xia Luo ${ }^{1 *}$, Xiang $\mathrm{Li}^{2}$
}

\begin{abstract}
Objective: To observe the curative effects of rh-endostatin combined with DP regimen in treating patients with advanced esophageal cancer and analyze the correlation of CT perfusion (CTP) parameters and the expression of vascular endothelial growth factor (VEGF). Methods: Twenty patients with esophageal cancer confirmed pathologically were randomly divided into combined treatment (rh-endostatin+DP regimen) group and single chemotherapy group, 10 patients in each group, respectively. All patients were given conventional CT examination and CTP imaging for primary tumor. The level of VEGF, the size of tumor and CTP parameters (BF, BV, PS and MTT) before treatment and after 2 cycles of treatment were determined for the comparison and the correlation between CTP parameters and VEGF expression was analyzed. Results: the therapeutic effect of rh-endostatin+DP regimen group was superior to single chemotherapy group. VEGF level after treatment in rhendostatin $+D P$ regimen group was obviously lower than single chemotherapy group $(P<0.01)$. The expression of VEGF had positive correlation with BF and BV but negative correlation with MTT. Compared with treatment before for rh-endostatin +DP regimen group, BF, BV and PS decreased while MTT increased after treatment $(P<0.05)$. However, there were no significant differences between treatment before and after treatment in single chemotherapy $(P>0.05)$. Conclusions: Rh-endostatin can down-regulate the expression of VEGF in esophageal cancer, change the state of hypertransfusion and high permeability of tumor vessels and had the better curative effect and slighter adverse reactions when combined with chemotherapy.
\end{abstract}

Keywords: Esophageal cancer - endostatin - CT perfusion - normalization

Asian Pac J Cancer Prev, 15 (16), 6565-6570

\section{Introduction}

Angiogenesis is the basis of the occurrence, development and metastasis of tumors which can grow fast and further invade the surrounding tissues and distant metastasis by entering blood circulation only after the formation of new vessels (Folkman, 2007). Vascular epithelial growth factor (VEGF) can be regarded as the target for inhibiting the growth of tumors and preventing tumor node metastasis (Andrea et al., 1999; Carmeliet et al., 2011). Endostatin is a kind of endogenous angiogenesis inhibiting factor, based on which recombinant human endostatin (rh-endostatin/endostar), a new artificially synthesized targeted drug for anti-angiogenesis, comes into being (Sun et al., 2007; Cao et al., 2011). Previous study has proven that rh-endostatin, which has synergistic effect when combined with chemotherapeutics, can improve the efficacy of chemotherapy for non-small cell lung cancer and has a good prospect of clinical application (Yang et al., 2005; Yang et al, . 2006). CT perfusion (CTP), as a technology of functional imaging marked by fast, accuracy and non-invasive benefits, is available for the evaluating the blood flow perfusion status of living tissues and organs. Therefore, CTPI was used to observe and analyze the efficacy of rh-endostatin combined with chemotherapy in treatment of 20 patients with advanced esophageal cancer from August, 2010 to May, 2012 and the results are as follows.

\section{Materials and Methods}

\section{Case selection}

Inclusion criteria: Patients with esophageal cancer confirmed by pathology and/or cytology; patients who had objective measurable primary lesions; stage IIIb IV before treatment according to criteria of Union for International Cancer Control (UICC) in 2009 (Chen, 2010); generally Eastern Cooperative Oncology Group (ECOG) scores are 0 2. The examination indexes confirm to chemotherapeutic indications; no dysfunction of primary organs; drug discontinuance for more than 4 weeks because of the history of use of other 
Table 1. Genral Data of Patients with Esophageal Cancer Between 2 Groups (n)

\begin{tabular}{|c|c|c|c|c|}
\hline \multirow{2}{*}{$\begin{array}{l}\text { Clinical data } \\
\text { Sex }\end{array}$} & \multicolumn{2}{|c|}{$\begin{array}{l}\text { Rh-endostatin+DP } \\
\text { regimen group }(n=8)\end{array}$} & \multirow[t]{2}{*}{$\begin{array}{l}\text { Single chemotherapy } \\
\text { group }(\mathrm{n}=10)\end{array}$} & \multirow{2}{*}{$\begin{array}{l}P \text { value } \\
\mathrm{P}>0.05\end{array}$} \\
\hline & & & & \\
\hline male & & 5 & 3 & \\
\hline female & & 3 & 7 & \\
\hline \multicolumn{2}{|c|}{ pathological pattern } & & & $P>0.05$ \\
\hline \multicolumn{2}{|c|}{ adenocarcinoma } & 0 & 0 & \\
\hline \multicolumn{2}{|c|}{ squamous carcinoma } & 8 & 10 & \\
\hline \multicolumn{2}{|c|}{ clinical stages } & & & $P>0.05$ \\
\hline \multicolumn{2}{|l|}{ IIIb } & 2 & 3 & \\
\hline \multicolumn{2}{|l|}{ IV } & 6 & 7 & \\
\hline \multicolumn{2}{|l|}{ ECOG score } & & & $P>0.05$ \\
\hline \multicolumn{2}{|l|}{$0 \sim 1$ score } & 7 & 9 & \\
\hline 2 score & & 1 & 1 & \\
\hline
\end{tabular}

chemotherapeutics; the expected survival time $\geq 3$ months; patients who were willing to accepted this treatment plan, with good compliance.

Exclusion criteria: CTP failures; patients who had no measurable tumor lesions; pregnant and lactating women; patients with metastatic tumor of central nervous system who didn't get effective control; patients who didn't complete the treatment course of chemotherapy; patients who were not performed with CT examination.

\section{General data}

A total of 20 patients from August, 2010 to May, 2012 were enrolled for forward-looking, randomized and controlled clinical trial and randomly divided into single chemotherapy group and rh-endostatin + DP regimen group according to the ratio of 1:1. However, 2 cases were excluded. Of which, one withdrew from the study after only completeness of 1-cycle treatment because of the presence of IVdegree of digestive tract reaction after treatment. The other withdrew from the study because of the presence of IVdegree of myelosuppression during the treatment course of the second cycle. Therefore, 18 cases can be evaluated, and 8 were in rh-endostatin + DP regimen group while 10 in single chemotherapy group. Age, sex, performance status, clinical stages, pathological pattern between 2 groups has good balance and comparability $(P>0.05)$ (Table 1$)$.

\section{Treatment methods}

Single chemotherapy group received DP regimen: intravenous drip of docetaxel and cisplatin on the first day of each cycle, $75 \mathrm{mg} / \mathrm{m} 2$, respectively, repeated every 21 d. Based on single chemotherapy plan, rh-endostatin+DP regimen group was given continuous intravenous drip of rh-endostatin for $14 \mathrm{~d}, 15 \mathrm{mg}$, repeated every $21 \mathrm{~d}$. The above plan was completed at least 2 cycles.

\section{VEGF detection method}

Venous blood $(10 \mathrm{~mL})$ was drawn before treatment and after treatment. Then enzyme-linked immunosorbent assay (ELISA) was employed to detect the content of VEGF. The above procedures were completed by supervising technician according to the instruction under strict quality control.

\section{CT perfusion scan method}

GE Light Speed 32-row CT scanner was applied with $120 \mathrm{Kv}, 150 \mathrm{mAs}, 5 \mathrm{~mm}$-thick layer, large scanning field and standard reconstruction method. Patients in supine position and under the state of eupnea were performed with conventional chest $\mathrm{CT}$ plain scan for determining the location and the largest layer of esophageal cancer, based on the center of which, 8 layers were selected as the scope of perfusion scan. $50 \mathrm{~mL}$ of contrast media (ioversol, 320 $\mathrm{mgl} / \mathrm{mL}$ ) was infused through elbow vein by high pressure injector, with injection speed being $4 \mathrm{~mL} / \mathrm{s}$. Then after the delay of $5 \mathrm{~s}$, the selected layers received continuous dynamic enhanced scan, with scanning time being $45 \mathrm{~s}$. The second examination was done for 2 groups under the same situation within $1 \sim 10 \mathrm{~d}$ after the completeness of the first cycle.

\section{CTP image post-processing}

CTP scanning primary images were deduced to Advantage Windows 4.3 (AW 4.3) work station, and analyzed by CT perfusion-3 tumor software package. Through the automatic processing of software package, color-coded graphs of time-desenty-curves (TDC), blood flow (BF), blood volume (BV), mean transit time (MTT), and permeability surface (PS) processed by contrast agent were obtained.

\section{Observational indexes}

The short-term efficacy of 2 groups was observed. Conventional CT examination and CTP imaging for primary tumor were performed. The level of VEGF, the size of tumor and CTP parameters (BF, BV, PS and MTT) before treatment and after 2 cycles of treatment were determined for the comparison and the correlation between CTP parameters and VEGF expression was analyzed.

\section{Evaluation criterion}

The size of tumor lesion was evaluated according to Response Evaluation Criteria in Solid Tumors (RECIST) (Yan, 2000) and divided into complete remission (CR), partial remission (PR), stability of disease (SD) and progression of disease $(\mathrm{PD})$. The effective rate $=\mathrm{CR}+\mathrm{PR}$, and total stability rate $=\mathrm{CR}+\mathrm{PR}+\mathrm{SD}$. $\mathrm{CT}$ examination was performed one week before chemotherapy and one week after the completeness of the second cycle. Security indexes were evaluated by WHO antitumor drug acute and subacute toxicity and grading standard (Sun, 2007).

\section{Statistical data analysis}

SPSS15.0 software was used for data analysis. $t$ test was for comparison of 2 groups and paired t test for the differences between treatment before and after treatment. The correlation between CT parameters and VEGF was analyzed by Pearson test. $P<0.05$ was considered to be statistically significant.

\section{Results}

Short-term efficacy and safety

Short-term efficacy: There was no CR in patients of 2 groups. Of 8 patients in rh-endostatin $+\mathrm{DP}$ regimen 
group, 4 cases were PR, 2 SD and 2 PD and remission rate was $50.0 \%(4 / 8)$, total stability rate was $75.0 \%(6 / 8)$; there were $3 \mathrm{PR}, 3 \mathrm{SD}, 4 \mathrm{PD}$ in single chemotherapy group and remission rate was 30\% (3/10), total stability rate was $60.0 \%(6 / 10)$. Remission rate and stability rate of rh-endostatin + DP regimen group were higher than single chemotherapy group but there were no significant differences $(P>0.05)$.

Safety evaluation: Adverse reactions of 20 patients can be evaluated and were mainly manifested with
A

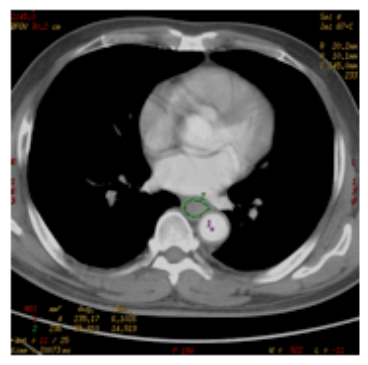

C

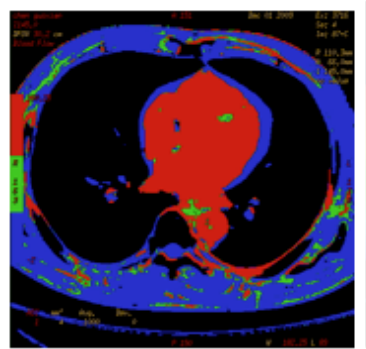

$\mathbf{E}$

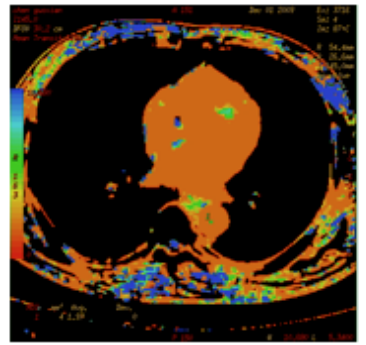

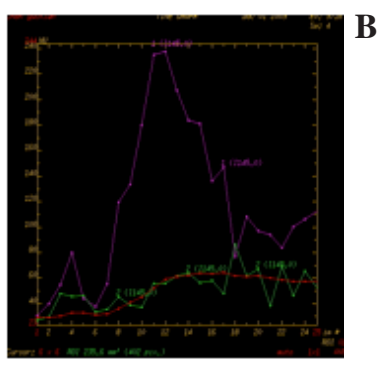
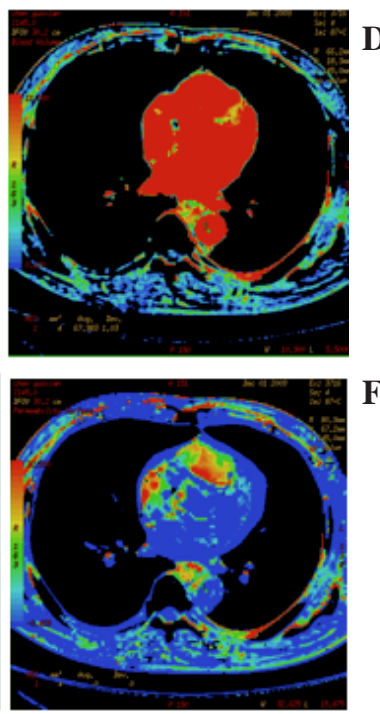

Figure 1. Esophageal Differentiated Squamous Carcinoma CTP Images. A) CTP ROI selected figure. " 1 " refers to the ROI of input artery; " 2 " refers to the ROI of tumor. B) Curve 1 (purple): the TDC of the ROI of tumor; curve 2 refers to the TDC of the ROI. C) BF figure. BF value is $(100.25 \pm 36.52) \mathrm{mL} / 100 \mathrm{~g} / \mathrm{min}$. Green color can be seen in the center and red in the margin of tumor, showing high blood flow in the margin and low blood flow in the center of tumor. D) BV figure. BV value is $(6.99 \pm 3.78) \mathrm{mL} / 100 \mathrm{~g}$. Red can be seen in the center and green in most of the margin of tumor, suggesting larger blood volume in the center of tumor. E) MMT figure. MTT value is $(8.38 \pm 3.81) \mathrm{s}$. Green can be seen in the center and orange in the margin, showing shorter MTT in the margin of tumor. F) PS figure. PS value is $(9.76 \pm 2.65) \mathrm{s}$. Red can be seen in a small part of the margin and green and yellow in the rest of area, indicating low vascular permeability of tumor while relatively high in the margin myelosuppression and gastrointestinal reaction, but there were no significant differences between two groups $(P>0.05)$. In addition, there were 4 cases of electrocardiogram (ECG) changes marked by $\mathrm{T}$ wave change, slighter degree of ST-segment depression and nodal tachycardia. One $\mathrm{T}$ wave change happened in single chemotherapy group and the other three happened in rh+endostatin plan group, but the symptoms returned to normal after drug discontinuance or symptomatic treatment.

\section{Comparison of the level of VEGF between 2 groups}

There were no statistical differences before treatment between 2 groups $(P>0.05)$. The level of VEGF after treatment in both 2 groups significantly decreased $(P<0.01)$. Rh-endostation +DP regimen group was obviously lower than single chemotherapy group $(P<0.05)$. (Table 2).

CTP parameters before treatment and after treatment between 2 groups

There were no statistical differences before treatment between 2 groups $(P>0.05)$. Compared wth before treatment, BF, BV and PS after treatment in rhendostatin $+\mathrm{DP}$ regimen group reduced and MTT increased $(P<0.05$, or $P<0.01)$. BF, BV and PS after treatment in single chemotherapy group decreased and MTT increased, but there were no significant differences $(P>0.05)$. There were statistical differences in BF, BV and PS after treatment between 2 groups $(P<0.05)$ but no differences in MTT $(P>0.05)$ (Table 3). Example of CTP pcolor was as shown in Figure 1.

Table 2. The Comparison of the Level of VEGF Between 2 Groups ( $\bar{x} \pm s)$

\begin{tabular}{lll}
\hline Group & \multicolumn{1}{c}{$\mathrm{n}$} & \multicolumn{1}{c}{ VEGF $(\mathrm{pg} / \mathrm{ml})$} \\
\hline rh-endostation + & Treatment before $(\mathrm{n}=10)$ & $646.58 \pm 100.75$ \\
DP regimen group & After treatment $(\mathrm{n}=8)$ & $485.34 \pm 95.23^{* * *}$ \\
single chemotherapy & Treatment before $(\mathrm{n}=10)$ & $658.13 \pm 105.66$ \\
group & After treatment $(\mathrm{n}=10)$ & $576.98 \pm 87.46^{* *}$ \\
\hline
\end{tabular}

Compared with treatment before, $* * P<0.01$; compared with single chemotherapy group, ${ }^{\#} P<0.05$

Table 4. The Correlation Between CTP Parameters and the Expression of VEGF

\begin{tabular}{lcl}
\hline & \multicolumn{2}{c}{ VEGF } \\
\cline { 2 - 3 } & $\mathrm{R}$ & $\mathrm{P}$ \\
\hline BF & 0.436 & $0.013^{*}$ \\
BV & $0.513^{*}$ & $0.041^{*}$ \\
MTT & -0.308 & $0.035^{*}$ \\
PS & 0.283 & 0.098 \\
\hline
\end{tabular}

$* P<0.05$

Table 3. CTP Parameters Before Treatment and After Treatment Between 2 Groups $(\overline{\mathrm{x}} \pm \mathrm{s})$

\begin{tabular}{llcccl}
\hline Group & & BF & BV & PS & MTT \\
\hline Rh-endostatin +DP regimen group & Treatment before $(\mathrm{n}=10)$ & $119.67 \pm 50.01$ & $7.88 \pm 2.09$ & $13.44 \pm 7.56$ & $6.08 \pm 2.74$ \\
& After treatment $(\mathrm{n}=8)$ & $71.01 \pm 45.33^{* \#}$ & $4.24 \pm 2.11 * * \#$ & $4.80 \pm 6.01 * \#$ & $8.95 \pm 2.30 *$ \\
Single chemotherapy group & Treatment before $(\mathrm{n}=10)$ & $113.53 \pm 41.46$ & $7.67 \pm 3.78$ & $12.87 \pm 8.52$ & $6.09 \pm 3.64$ \\
& After treatment $(\mathrm{n}=10)$ & $105.45 \pm 20.76$ & $6.88 \pm 2.96$ & $11.44 \pm 7.02$ & $7.48 \pm 2.56$ \\
\hline
\end{tabular}

Notes: BV unit: $\mathrm{ml} / 100 \mathrm{~g}$; BF, PS: $\mathrm{ml} /(\mathrm{min} \cdot 100 \mathrm{~g})$; MTT: s; Compared with treatment before, ${ }^{*} P<0.05$, or $* * P<0.01$; Compared with single chemotherapy group, ${ }^{\sharp} P<0.05$ 
The correlation between CTP parameters and the expression of VEGF

Pearson analysis showed the expression of VEGF had positive correlation with $\mathrm{BF}$ and $\mathrm{BV}$ but negative correlation with MTT $(P<0.05)$. PS had no correlation with the expression of VEGF $(P>0.05)$ (Table 4$)$.

\section{Discussion}

At present, esophageal cancer ranks the 8th of the incidence of malignant tumors all over the world and patients with esophageal cancer in China takes up more than $50 \%$ of the world, with the mobility and cancer related mortality ranking the 4th, highly occurring in Lin County and Anyang City, Hennan Province (Yuan et al., 2014; Malik et al., 2014; Yang et al., 2014; Somi et al., 2014; Zheng et al., 2014). About $50 \%$ of patients were found with the advanced period of esophageal cancer when diagnosed, with unfavorable prognosis, about 6 8 months of natural course, $5 \% \sim 7 \%$ five-year survival rate (; Liu et al., 2014; Mai et al., 2014; Zhao et al., 2014; Jiang et al., 2014; Wang et al., 2014; Stefani et al., 2014). Additionally, even though patients accepted surgical treatment, there still exists postoperative recurrence and metastasis in nearly $90 \%$ of patients. $50 \%$ of patients in early stage recurs within 5 years and the autopsy confirmed that there appeared distant metastasis in $50 \%$ patients with local advanced esophageal cancer in the clinic (Liu et al., 2009). Therefore, chemotherapy plays an important role in the treatment of esophageal cancer, with the intention to control the metastasis of tumor. In last decade, largescale randomized controlled trials about esophageal cancer have proved that chemotherapy could improve long-term 5-year survival rate and targeting therapy started the new age of the treatment of esophageal cancer.

Domestic and foreign researches proved that tumor angiogenesis played an important role in the occurrence, development, surrounding infiltration, lymphatic metastasis and distant metastasis of solid tumor, markedly influences the treatment and prognosis of patients with tumors, so blocking the formation of tumor blood vessels can slow down the speed of the growth of tumor and improve the prognosis of the patients (Mizobuchi et al., 2008). Since $1993 \mathrm{Kim}$ found blocking the expression of VEGF acting in blood vascular system could significantly inhibit the growth of tumor, an increasing number of researches showed that the level of VEGF reflexed the proliferation, migration and blood vessel construction of tumor endothelial cell and directly reflected the growth speed and metastasis of tumor (Bondestam et al., 2000). Shan et al (Shan et al., 2004) suggested that the positive expression rate of VEGF in esophageal cancer tissues was $70.9 \%$ and the expression of VEGF was associated with pathology classification, depth of invasion and lymphatic metastasis, which provide the theoretical foundation for the treatment of esophageal cancer by blocking the expression of VEGF. The related studies were reported abroad (Inoue et al., 1997; Kozlowski et al., 2010)

$\mathrm{Rh}$-endostatin is recombinant human endostatin with multiple target points, developed by our country and its mechanism is to influence the transduction pathway of
VEGF and the activity of proteolytic enzyme, inhibit the migration of endothelial cells and induce the cell apoptosis, thus achieving the purpose of anti-tumor angiogenesis (Wang et al., 2003; Liang et al., 2005). During the course of treatment, rh-endostatin may play an important role in inhibiting angiogenesis which gives rise to the inhibition of the growth of tumor because of ischemia and anoxemia of tumor tissues during the course of inhibiting angiogenesis. Moreover, rh-endostatin can down-regulate the level of MMP-7 and MMT-14 by rhendostatin inhibiting the level of VEGF. Experiments have revealed that rh-endostatin, which is more widely used, has multiple effects on anti-tumor (Shao et al., 2014; Feng, 2014). Huang et al (Huang et al., 2009) reported a randomized study of Shenyi Capsule plus gemcitabine combined with cis-platinum in the treatment of advanced esophageal cancer and found there were no significantly differences in overall response rate between 2 groups, but combined group was obviously lower than control group in the level of VEGF after treatment $(P<0.05)$, which was consistent with the results of this study. This study showed that remission rate and stability rate of rh-endostatin +DP regimen group were higher than single chemotherapy group but there were no significant differences $(P>0.05)$. The level of VEGF after treatment in both 2 groups significantly decreased $(P<0.01)$. rhendostation $+\mathrm{DP}$ regimen group was obviously lower than single chemotherapy group $(P<0.05)$.

Response evaluation criteria in solid tumors (RECIST) is currently the general international standard for evaluating the efficacy of solid tumor. It used the maximum diameter instead of double path. The most content excerpts from revised tumor efficacy judgment standard made by WHO. hypoxic cell, necrotic tissue, non-cellular constituent (such as tumor stroma), which account for most of tumor in volume, are poorly sensitive to chemotherapy while active tissue of tumor takes up small part of the volume of tumor and get smaller as the tumor become bigger. Therefore, when the volume of tumor shrinks through radiology and chemotherapy, the actual number of active cells which are killed could exceed the proportion. Similarly, when the tumor grows bigger, the actual number of active cells increases many times, even more. Therefore, based on RECIST, the judgment is not consistent with clinical fact.

Therefore, the condition of tumor angiogenesis before treatment and after treatment is the important index of molecular biology for judging the activity of tumor. At present, in clinical practice, micro-vessel density (MVD) and VEGF in biopsy tissue of tumor are commonly used to reflex the state of angiogenesis. However, they don't become the ideal examination methods because of their traumatic, separated defects and non-functional evaluation. If the prognosis can be evaluated through certain methods before the examination, the proper treatment method will be chosen timely for clinical application, consequently relieving the pain and the financial burden of the patients and reduce waste of medical resources. imaging method is used to conduct vascular quantitative and functional examination in recent year and detecting instruments includes color doppler ultrasound, CT, magnetic resonance 
and PET-CT. CT perfusion imaging (CTPI) is considered as a functional imaging technique which can evaluate microcirculation condition of living tissues, reflect physiological and metabolic changes of tissues and organs and reflex the clinical features before tumor morphology do not change or change a little. Blood supply of tumor is used to be the predictive index for evaluating the efficacy of the treatment of tumor, which provide the new methods for clinical diagnosis and treatment of tumor.

Tumor blood vessels were manifested as high perfusion and high permeability in CTPI. Compared with normal tissue, BV, BF, PS and MTT increased but "vascular normalization" after treated with chemotherapeutics and anti-angiogenesis drugs was manifested as decreased PS, BF and BV and increased MTT (Zhang et al., 2006). CTP parameters analysis in another study related to esophageal cancer showed that BF, BV and PS after chemotherapy decreased more obviously than treatment before (Bellomi et al., 2007; Wu et al., 2009). In this study, Compared wth before treatment, BF, BV and PS after treatment in rhendostatin $+\mathrm{DP}$ regimen group reduced and MTT increased $(P<0.05$, or $P<0.01)$ and there were statistical differences in BF, BV and PS after treatment between 2 groups $(P<0.05)$, showing the feature of "vascular normalization".

Correlation analysis in this study showed that BV and $\mathrm{BF}$ had positive correlation with the expression of VEGF while MTT had negative correlation with the expression of VEGF. BV can reflect dynamic infusion state and blood volume of blood flow in local tissues, so with the increase of VEGF in tissue of esophageal cancer, MVD and blood circulation increased and $\mathrm{BV}$ value also rise, which showing BV had positive correlation with the expression of VEGF. BF value of CTP increased in the early stage, but decreased gradually with the progression of the treatment, the expression of VEGF also down-regulated.

In conclusion, rh-endostatin can enhance the efficacy of patients with esophageal cancer, reduce the adverse reactions and change the state of high perfusion and high permeability of tumor vessels. Further study can be still done to analyze the relation between survival rate and the changes of CTP parameters through using other imaging methods (such as PETCT or dynamic MRI control), long-term followup, and large-scale sample as well as new standard for judging the clinical efficacy.

\section{References}

Andrea K, Krin W, Andreas O, et al (1999). Vascular endothelial growth factor in the sera and effusions of patients with malignant and nonmalignant disease. Cancer, 85, 178-87.

Bondestam J, Salven P, Jääskelä-Saari H, et al (2000). Major surgery increases serum levels of vascular endothelial growth factor only temporarily. Am J Surg, 179, 57-9.

Bellomi M, Petralia G, Sonzogni A, et al (2007). CT perfusion for the monitoring of neoadjuvant chemotherapy and radiation therapy in rectal carcinoma: initial experience. Radiology, 244, 486-93.

Chen LQ (2010). International TND installment interpretation and evaluation for esophageal cancer. Chin J Oncol, 32,
237-40.

Cao DD, Ge W, Wang HM, ea al (2011). Efficacy and Safety of rh-endosratin combined with chemotherapy versus chemotherapy alone for asvanced NSCLC, a metaoanalysis review. Chin J Lung Cancer, 14, 404-13.

Carmeliet P, Jain RK (2011). Principles and mechanisms of vessel normalization for cancer and other angiogenic diseases. Nat Rev Drug Discov, 10, 417-27.

Folkman J (2007). Angiogenesis, an organizing principle for drug discovery? Nat Rev Drug Discov, 6, 273-86.

Huang JY1, Sun Y, Fan QX, Zhang YQ (2009). Efficacy of Shenyi Capsule combined with gemcitabine plus cisplatin in treatment of advanced esophageal cancer, a randomized control trial. Zhong Xi Yi Jie He Xue Bao, 7, 1047-51.

Inoue K1, Ozeki Y, Suganuma T, Sugiura Y, Tanaka S (1997). Vascular endothelial growth factor expression in primary esophageal squamous cell carcinoma. Cancer, 79, 206-13.

Kozlowski M, Naumnik W, Niklinski J, et al (2010). Vascular endothelial growth factor $\mathrm{C}$ and $\mathrm{D}$ expression correlates with lymph node metastasis and poor prognosis in patients with resected esophageal cancer. Neoplasma, 58, 311-9.

Jiang C, Liao FX, Rong YM, et al (2014). Efficacy of TaxaneBased Regimens in a First-line Setting for Recurrent and/or Metastatic Chinese Patients with Esophageal Cancer. Asian Pac J Cancer Prev, 15, 5493-8.

Liang JF, Pan MY (2005). Research progress of endostatin. Milit Med J Southeast China, 7, 475-8.

Liu JL, Zhou LL, Cai MH, et al (2009). Observation on the short-term effect of nedaplatin plus tegafur in the treatment of advanced esophageal carcinoma. J Pract Oncol, 24, 515-6.

Liu C, Jiang Z, Deng QX, et al (2014). Meta-analysis of Association Studies of CYP1A1 Genetic Polymorphisms with Digestive Tract Cancer Susceptibility in Chinese. Asian Pac J Cancer Prev, 15, 4689-95.

Mai RQ, Huang B, Shen L, et al (2014). Genotype distribution of human papillomavirus in women with abnormal cervical cytology in an esophageal carcinoma high incidence area of china. Asian Pac J Cancer Prev, 15, 4945-50.

Malik MA, Umar M, Gupta U, et al (2014). Phospholipase C epsilon 1 (PLCE1 rs2274223A\&gt;G, rs3765524C\&gt; T and rs7922612C\&gt;T) polymorphisms and esophageal cancer risk in the Kashmir Valley. Asian Pac J Cancer Prev, 15, 4319-23.

Mizobuchi H, García-Castellano JM, Philip S, et al (2008). Hypoxia markers in human osteosarcoma, an exploratory study. Clin Orthop Relat Res, 466, 2052-9.

Shan BE, Pan LF, Li QX, et al (2005). Study on the expression of VEGF in human esophageal cancer tissue. Cancer Res Prev Treat, 32, 644-7.

Somi MH, Golzari M, Farhang S, et al (2014). Gastrointestinal cancer incidence in East Azerbaijan, Iran, update on 5 year incidence and trends. Asian Pac J Cancer Prev, 15, 3945-9.

Stefani ED, Boffetta P, Ronco AL, et al (2014). Processed meat consumption and squamous cell carcinoma of the oesophagus in a large case-control study in uruguay. Asian Pac J Cancer Prev, 15, 5829-33.

Sun S, Schiller JH (2007). Angiogenesis inhibitor in the treatment of lung cancer. Crit Rev Oncol Hematol, 62, 93-104.

Sun Y, Shi KY (2007). Manual Medical Oncology. People's Medical Publishing House, 7, 135-45.

Shao MW, Qiu JR, Wu H, et al (2014). Mechanism of Shenyi Capsule Concomitant with Endostar and Chemotherapy on the Growth and Apoptosis of MCF-7 Breast Cancer Cells. J Int Transl Med, 2, 299-302.

Wang J, Yu JM, Jing SW, et al (2014). Relationship between EGFR Over-expression and Clinicopathologic Characteristics in Squamous Cell Carcinoma of the Esophagus, A Meta- 
analysis. Asian Pac J Cancer Prev, 15, 5889-93.

Wang JW, Yang L (2003). Endostatin and Advances in Its Clinical Research. J Clin Res, 20, 778-81.

Wu J, Long Q, Xu S, et al (2009). Study of tumor blood perfusion and its variation due to vascular normalization by anti-angiogenic therapy based on 3D angiogenic microvasculature. J Biomech, 42, 712-21.

Yan J, Zeng YX (2000). The latest guideline for response evaluation criteria in solid tumors. Foreign Med Sci (Cancer Section), 27, 375-6.

Yang L, Wang JW, Cui XC (2005). Rh-endostatin (YH-16) in combination with vinorebine and cisplatin for advanced non-small cell lung cancer, a multicenter phase IItrial. Chin J New Drugs, 14, 204-7.

Yang L, Wang JW, Sun Y, et al (2006). Ramdomized phase IItrial on escalated doses of rhendosstatin ( $\mathrm{YH}-16)$ for advanced non-small cell lung cancer. Chin J Oncol, 28, 138-41.

Yang JZ, Ji AF, Wang JS, et al (2014). Association between Ras association domain family $1 \mathrm{~A}$ promoter methylation and esophageal squamous cell carcinoma, a meta-analysis. Asian Pac J Cancer Prev, 15, 3921-5.

Yuan P, Chen TH, Chen ZW, et al (2014). Calculation of life-time death probability due malignant tumors based on a sampling survey area in China. Asian Pac J Cancer Prev, 15, 4307-9.

Zhang JE, Liang CH, Zhao ZJ, et al (2006). Utility of CT perfusion in pulmonary nodules. Chin J Radiol, 39, 1041-5.

Zhao L, Wei ZB, Yang CQ, et al (2014). Effects of PLCE1 Gene Silencing by RNA Interference on Cell Cycling and Apoptosis in Esophageal Carcinoma Cells. Asian Pac J Cancer Prev, 15, 5437-42.

Zheng NG, Wang JL, Yang SL, et al (2014). Aberrant Epigenetic Alteration in Eca9706 Cells Modulated by Nanoliposomal Quercetin Combined with Butyrate Mediated via EpigeneticNF- $x$ B Signaling. Asian Pac J Cancer Prev, 15, 4539-43. 